\title{
Fluoroquinolones resistant Gram-positive cocci isolated from University of Calabar Teaching Hospital, Nigeria
}

\author{
Unimke Augustine A. * and Andy Iniobong E. \\ Department of Microbiology, Faculty of Biological Sciences, University of Calabar, PMB 1115, Calabar, Cross River State, \\ Nigeria.
}

Publication history: Received on 18 August 2017; revised on 05 October 2017; accepted on 09 October 2017

https://doi.org/10.30574/gscbps.2017.1.1.0003

\begin{abstract}
Eighty four clinical samples of Gram-positive cocci isolated from University of Calabar Teaching Hospital, Nigeria were examined for resistance to fluoroquinolones antibiotics using disc diffusion method on Muller Hinton agar. Forty four of the total isolates were Staphylococcus spp. while forty isolates were Streptococcus spp. Resistance rates were highest against ofloxacin 18 (41\%) for Staphylococcus spp. and 10 (25\%) for Streptococcus spp. followed by sparfloxacin 15 (34\%) and 6 (15\%) for Staphylococcus spp. and Streptococcus spp., respectively; while resistance to ciprofloxacin was the least $14(32 \%)$ and 4 (10\%) for Staphylococcus spp. and Streptococcus spp., respectively. These findings highlight the development of resistance by Gram-positive cocci to fluoroquinolones and suggest for the careful and appropriate administration of these agents as well as infection-control practices.
\end{abstract}

Keywords: Fluoroquinolones; Gram-positive cocci; Antibiotic resistance; Staphylococcus; Streptococcus

\section{Introduction}

The development of antibiotic resistance by bacteria is an evolutionary certainty, because of their ability to adapt to unfavorable ecological conditions. Fluoroquinolones are medications that kill bacteria or prevent their growth [1-2]. Resistance to fluoroquinolones among Gram-positive cocci has emerged as these antimicrobial agents have become extensively used in clinical medicine. Resistance is effected via changes in the bacterial target enzyme DNA gyrase and topoisomerase IV which reduce drug binding, and through movement of native bacterial membrane pumps to remove drug from the cell. In both cases, quinolones exposure selects for spontaneous mutants such as chromosomal mutations which alter the target protein or increase the level of pump expression [3-4].

When quinolones are used to treat infections caused by other bacterial pathogens, subject colonized with staphylococcus aureus (e.g., on their skin or mucosal surfaces) are likely exposed to sub-therapeutic antibiotic concentration and are therefore at risk becoming colonized with resistant mutants. These resident resistant strains then become the reservoir for future infections [4].

Antibiotic resistance in Gram-positive cocci is one of the major public health concerns for the beginning of the $21^{\text {st }}$ century [2]. The pharmaceutical industry has responded with the development of oxalidinones, lipopeptides, injectables, streptomycins, ketolides, glycylcyclines, second generation glycopeptides and novel fluoroquinolones [56]. However, clinical use of these novel agents will cause new selective pressures and will continue to drive the development of resistance.

\footnotetext{
*Corresponding author

E-mail address: austinraney.unimke@ gmail.com
} 


\section{Material and methods}

\subsection{Materials}

The materials employed in this study were obtained from reputable scientific outlets (Difco Laboratories, Michigan) and were stored according to their manufacturer's instructions until required for used.

\subsubsection{Study site}

This research was carried out in the Microbiology Research Laboratory, University of Calabar, Nigeria.

\subsubsection{Study samples}

The samples employed in this study were Staphylococcus spp. and Streptococcus spp. isolated from different sources at the University of Calabar Teaching Hospital, Nigeria

\subsubsection{Culture media}

The culture media employed were nutrient agar, blood agar, MacConkey agar, and Muller Hinton agar. They were all obtained in dehydrated powder form and stored at room temperature until required for use. All media were prepared according to the manufacturer's instruction [7].

\subsection{Methods}

Standard microbiological methods were employed in this study. The samples were cultured in different media. Biochemical tests were carried out for confirmation and disk diffusion technique was used in the sensitivity test according to specifications [8-10].

\subsubsection{Sample collection}

The samples were aseptically collected from patients in University of Calabar Teaching Hospital from different sources. The samples were principally collected by the medical laboratory scientist from; skin, burns, wounds, throat washing, aspirates, sputum and blood and all samples were taken immediately to the laboratory for culturing following laid down procedures as described by Hooper [4].

\subsubsection{Isolation of Gram-positive cocci}

The microorganisms were isolated from the clinical specimen onto solid media by streak plate method. The media employed were nutrient agar, blood agar and MacConkey agar [4, 11-12].

\subsubsection{Identification and confirmation of Gram-positive cocci}

All microorganisms growing on the various media were characterized on the basis of gram stain reaction, microscopy, catalase and coagulase tests. The identification of each organism was based on the results of the tests. The test results were evaluated using characteristics presented in Bergey's Manual of Determinative Bacteriology [13].

\subsubsection{Preservation of isolates}

The identified isolates of Streptococcus spp. and Staphylococcus spp. were preserved in nutrient Agar slant using bijou bottles. These bottles were properly labeled for easy identification. If not ready for use, they were stored in a refrigerator at $4{ }^{\circ} \mathrm{C}$; since high temperature will cause profound proliferation of the isolates progeny, which was not necessary for the work [11-12].

\subsubsection{Fluoroquinolones sensitivity test}

This test was carried out to determine the susceptibility and resistance of some fluoroquinolones (ciprofloxacin, sparfloxacin and ofloxacin) on gram-positive cocci (Staphylococcus spp. and Streptococcus spp.). In this work, a disk diffusion technique was employed [14].

The discs used in this research were laboratory prepared disc each with a concentration of $30 \mu \mathrm{g}$. Generally, three kinds of fluoroquinolones were used; ciprofloxacin (CPX) $500 \mathrm{mg}$, sparfloxacin (LM) $200 \mathrm{mg}$ and ofloxacin (OFL) 200 mg. They were obtained at Karmel pharmacy, 90B Goldie street Calabar Nigeria. Each of these antibiotics was used to prepare a set of 200 sensitive discs. 
Before preparation, the discs were separated and spread out singly in a foil paper and labeled respectively. The discs were divided into 6 and were placed into sterile bottles each containing 100 discs, which was sterilized in the oven at $160{ }^{\circ} \mathrm{C}$ for $1 \mathrm{~h}$ and allowed to cool. The discs were then stored in sterile capped bottles at temperature of $4{ }^{\circ} \mathrm{C}$. The dilution used for each antibiotic was dependent on the concentration of the antibiotics most suitable for the work. A control disc was used. A loopful of 2-5 discrete colonies of purified test microorganisms were inoculated into 5 ml sterile nutrient broth. The inoculated broth culture was incubated overnight and then diluted to have approximately $10^{5}$ organism/ ml.

The diluted broth was used to flood the entire surface of the sensitivity agar (Muller Hinton Agar) plates. Excess broth was remove by draining it aseptically into a beaker of disinfectant and the plates dried, using a sterile pair of forceps, appropriate antimicrobial discs were firmly placed onto the surface of the plate about $15 \mathrm{~mm}$ from the edge of the plate and $25 \mathrm{~mm}$ from disc to disc with each tightly pressed down to ensure contact with the agar. The plates were left at room temperature for about an hour to allow for diffusion of the antimicrobial agents. The plates were incubated overnight at $37{ }^{\circ} \mathrm{C}$. After overnight incubation, the plates were examined for zones of inhibition. The zone diameters were measured using a transparent ruler in mm and compared with those of the control organism [5-6, 8-9, 15-17].

\section{Results and discussion}

The genus Staphylococci has at least 40 species. The three species used in this research were the most frequently encountered species of medical importance which are Staphylococcus aureus, Staphylococcus epidermidis, and Staphylococcus saprophyticus. The Streptococci are a large and heterogeneous group of bacteria and no one system suffices to classify them. The species employed in this research were Streptococcus pyogenes, Streptococcus agalactiae, and Streptococcus pneumonia. A total of 84 samples were collected from different sources at the University of Calabar Teaching Hospital, Nigeria. Out of the 84 samples collected, 44 were Staphylococcus spp. and 40 were Streptococcus spp.

This research has shown that fluoroquinolones are very active against Gram-positive cocci with ciprofloxacin 30 (68\%) being the most active member on Staphylococcus spp. and sparfloxacin 29 (59\%) is the second most active antibiotic in the group, while Ofloxacin $26(59 \%)$ has limited activity on Staphylococcus spp. This susceptibility rate is low when compared with a research work carried out by Franklin [6] on antimicrobial resistance: the example of Staphylococcus aureus at the University of Chicago USA who employed non-clinical samples. The resistance pattern of Gram-positive cocci was observed in the study. Staphylococcus spp. were mostly resistant to ofloxacin 18 (41\%) while ciprofloxacin 14 (32\%) and sparfloxacin 15 (34\%) were less resistant (Table 1). This finding is in agreement with reports of previous studies in this area by Blondeau [5] at the University of California, and Hooper [4] at the Oxford University College of Medicine.

Table 1 Mean sensitivity of Staphylococcus species to fluoroquinolones

\begin{tabular}{lcc}
\hline Antibiotics & Number of sensitive & Number of resistant \\
\hline Ciprofloxacin & $30(60 \%)$ & $14(32 \%)$ \\
Sparfloxacin & $29(66 \%)$ & $15(34 \%)$ \\
Ofloxacin & $26(59 \%)$ & $18(41 \%)$ \\
\hline
\end{tabular}

The sensitivity test carried out on Streptococcus spp. reveals that the isolates were highly sensitive to fluoroquinolones: ciprofloxacin 36 (90\%), sparfloxacin 34 (85\%) and ofloxacin 30 (75\%). Minimal resistance was observed among the isolates: ciprofloxacin 4 (10\%), sparfloxacin $6(15 \%)$ and ofloxacin 10 (25\%). This supports the findings of Bisno [1] who reported that Streptococcus spp. is highly susceptible to fluoroquinolones.

Table 2 Mean sensitivity of Streptococcus species to fluoroquinolones

\begin{tabular}{lcc}
\hline Antibiotics & Number of sensitive & Number of resistant \\
\hline Ciprofloxacin & $36(90 \%)$ & $4(10 \%)$ \\
Sparfloxacin & $34(85 \%)$ & $6(15 \%)$ \\
Ofloxacin & $30(75 \%)$ & $10(25 \%)$ \\
\hline
\end{tabular}


Table 3 reveals the cumulative sensitivity profile of Gram-positive cocci to fluoroquinolones. Significantly important is the low level of bacterial resistance associated with fluoroquinolones used (ciprofloxacin, sparfloxacin and ofloxacin) making it an essential treatment in critical situations. The resistance profile of some Staphylococcus spp. and Streptococcus spp. as observed in this study is in accordance with the findings of Lipsky who reported that since the emergence of penicillinase-producing Staphylococcus aureus in the 1940s, Staphylococci, Enterococci and Streptococci have proved themselves as multi drug resistance micro-organisms. [9]. The findings in this study of a high-level inhibition against Gram-positive cocci as observed in the cumulative susceptibility profile (Table 3) strictly conform to the work of Walker who reported that Gram-positive cocci may show a greater level of inhibition to fluoroquinolones $[14]$.

Table 3 Cumulative sensitivity profile of Gram-positive cocci to fluoroquinolones

\begin{tabular}{lcc}
\hline Microorganisms & Number of sensitive & Number of resistant \\
\hline Staphylococcus spp. & $28(64 \%)$ & $16(36 \%)$ \\
Streptococcus spp. & $33(83 \%)$ & $7(17 \%)$ \\
\hline
\end{tabular}

Mark et al. documented that fluoroquinolones exhibit a high level of antibacterial activity in-vitro and in-vivo and showed that although the newer agents offer a much broader spectrum of action, ciprofloxacin remains the most effective fluoroquinolones against Gram-positive and Gram-negative pathogens after researching on the prevalence of quinolones resistance among Gram-positive and Gram-negative pathogens at the University of Calabar Teaching Hospital [8]. The susceptibility and resistance profile shows that clinical isolates are more resistant to fluoroquinolones than non-clinical isolates for the reason being that clinical isolates are predisposed to several antimicrobial agents.

\section{Conclusion}

This study enables us to evaluate the antimicrobial activity of these antibiotics on Gram-positive cocci. For the result obtained, fluoroquinolones (ciprofloxacin, sparfloxacin and ofloxacin) have high activity against Gram-positive cocci, while minimal resistance was observed. This study also reveals that ciprofloxacin is the most active fluoroquinolone against Gram-positive cocci followed by sparfloxacin, while ofloxacin has limited activity.

\section{Compliance with ethical standards}

\section{Acknowledgments}

UAA sincerely acknowledge the immense contributions of all authors.

Disclosure of conflict of interest

Authors have declared that no competing interests exist.

\section{References}

[1] Bisno AL. (2004). Medical Progress: Group A Streptococcal infections and acute rheumatic fever. New England Journal of Medicine, 325, 783-793.

[2] Woodford NA. (2007). Biological counterstrike: Antibiotic resistance mechanisms of Gram- positive cocci. Journal of Infections and Health Protection, 12, 1123-1142.

[3] Foster TJ. (2006). Potential for vaccination against infections caused by Staphylococcus aureus. Vaccine, 9, 221228.

[4] Hooper DC. (2000). New use for new and old fluoroquinolones and the challenges of resistance. Clinical Infectious Diseases, 30, 24-32.

[5] Blondeau JM. (2009). Expanded activity and utility of the new fluoroquinolones. A Review of Clinical Therapy, 21, 3-7. 
[6] Franklin DL. (2003). Antimicrobial Resistance: the example of Staphylococcus aureus. Journal of Clinical Investigations, 9, 1265-1273.

[7] DIFCO (1984). Dehydrated culture media and reagents for microbiology (10 $10^{\text {th }}$ ed). Difco laboratories, Michigan.

[8] Mark HB, Beers MD and Berkow R. (2009). Antibacterial Drugs: Fluoroquinolones. The Merck Manual of Diagnosis and Therapy, 13, 153-174.

[9] Lipsky BA. (2000). Fluoroquinolones toxicity profiles. A review focusing on newer agents. Clinical Infectious Diseases, 28, 352.

[10] Yagupsky P. (2009). Selection of antibiotic-resistant pathogens in the community. Pediatric Infectious Disease Journal, 25, 974-976.

[11] Bhakdi SE and Tranum JJ. (2011). Alpha-toxin of Staphylococcus aureus. Microbial Reviews, 55, 733-741.

[12] Cambau ET. (2003). Mechanism of resistance to quinolones. Drugs, 45 (3), 15-19.

[13] Holt JG, Krieg NR, Senath PHA, Staley JT and Williams ST. (1994). Bergey's manual of determinative bacteriology. ( $9^{\text {th }}$ ed).Williams and Wilkins, Baltimore.

[14] Walker RC. (2011). The fluoroquinolones. Mayo Clinical Procedures, 74, 1030.

[15] McCracken GE. (2014). Emergence of resistant Streptococcus pneumonia: A problem in pediatrics. Pediatric Infectious Disease Journal, 14, 424 - 428.

[16] Giamarellou H. (2011). Activity of quinolones against Gram-positive cocci: Clinical features. Drug, 49 (2), 58-70.

[17] Kollef MH. (2007). Bugging the bugs: Novel approaches in the strategic management of resistant Staphylococcus aureus infections. Clinical Infectious Diseases, 45 (3), 163-164.

\section{How to cite this article}

Unimke AA and Andy IE. (2017). Fluoroquinolones resistant Gram-positive cocci isolated from University of Calabar Teaching Hospital, Nigeria. GSC Biological and Pharmaceutical Sciences, 1(1), 01-05. 